\title{
SOME FIXED POINT THEOREMS RELATING TO THE ORBITAL CONTINUITY
}

\author{
G. V. R. BABU AND M. V. R. KAMESWARI
}

\begin{abstract}
In this paper, we prove a fixed point theorem for asymptotically regular mappings on a metric space using orbital continuity of the selfmap. As an application of this result, a fixed point theorem is established in $T$-orbitally complete metric spaces. Our results extend Mukherjee's theorem [4] to $T$-orbitally complete metric spaces, and generalize the theorems of Jotic [5] and Nešić [6].
\end{abstract}

\section{Introduction}

Browder and petryshyn [2] introduced the concept of asymptotic regularity of a selfmap at a point in the space.

Definition 1.1. Let $(X, d)$ be a metric space, $T$ be a selfmap of $X$. $T$ is said to be asymptotically regular at a point $x$ in $X[2]$ if

$$
\lim _{n \rightarrow \infty} d\left(T^{n} x, T^{n+1} x\right)=0 .
$$

Let $x_{0} \in X ; O\left(x_{0}\right)=\left\{T^{n} x_{0}: n=0,1,2, \ldots\right\}$ is called the orbit of $x_{0}$.

Definition 1.2. A space $X$ is said to be T-orbitally complete [3] iff every Cauchy sequence which is contained in $O(x)$ for some $x$ in $X$ converges in $X$.

We observe that every complete metric space is $T$-orbitally complete for any $T$; but a $T$-orbitally complete metric space need not be a complete space.

Definition 1.3. $T$ is said to be orbitally continuous at a point $z$ in $X$ [1] if for any sequence $\left\{x_{n}\right\} \subset O(x)$ for some $x \in X, x_{n} \rightarrow z$ as $n \rightarrow \infty$ implies $T x_{n} \rightarrow T z$ as $n \rightarrow \infty$.

Clearly, any continuous mapping of a metric space is orbitally continuous, but its converse need not be true.

In 1995, Jotic [5] proved the following fixed point theorem in complete metric spaces.

Received September 30, 2003; revised March 08, 2004.

2000 Mathematics Subject Classification. 47H10, 54H25.

Key words and phrases. Selfmaps, fixed points, asymptotically regular maps, orbitally continuous mappings, $T$-orbitally complete metric space. 
Theorem 1.4. Let $(X, d)$ be a complete metric space, $T: X \rightarrow X$ a selfmap and $\varphi: R^{+} \rightarrow R^{+}\left(R^{+}=[0, \infty)\right)$ such that

$$
\begin{gathered}
\varphi(r)<r \quad \text { for } r>0 \\
\limsup _{t \rightarrow r+} \varphi(t)<r \quad \text { for } r>0 .
\end{gathered}
$$

If $T: X \rightarrow X$ satisfies the condition

$$
d\left(T x, T^{2} x\right) \leq \varphi(d(x, T x)) \text { for all } x \in X,
$$

then $\left\{T^{n} x\right\}$ is a Cauchy sequence. Furthermore, if $X$ is complete and if a mapping $G(x)=d(x, f(x))$ is lower semicontinuous at a limit point of $\left\{T^{n} x\right\}$, say $x^{*}$, then $x^{*}$ is a fixed point of $T$.

The following example shows that $T$ and $\varphi$ satisfying all the hypotheses of Theorem 1.4 , but the sequence $\left\{T^{n} x_{0}\right\}$ for any $x_{0} \in X$ is not Cauchy.

Example 1.5. Let

$$
X=\left\{\sum_{k=1}^{n} \frac{1}{k}: n=1,2,3, \ldots\right\}
$$

with the usual metric. We define $T$ on $X$ by

$$
T\left(\sum_{k=1}^{n} \frac{1}{k}\right)=\sum_{k=1}^{n+1} \frac{1}{k} .
$$

We define $\varphi: R^{+} \rightarrow R^{+}$by $\varphi(t)=t(1+t)^{-1}$ for $t>0$.

Then $T$ and $\varphi$ satisfying all the conditions of Theorem 1.4 and the sequence $\left\{x_{n}\right\}$ defined by $T x_{n}=x_{n+1}$ with $x_{0}=1$ is not Cauchy in $X$. Also, we observe that $T$ is asymptotically regular at $x_{0}$.

In fact in 1977, Mukherjee [4] proved the following theorem.

Theorem 1.6. Let $T$ be a selfmap of a complete metric space $X$. We suppose that there exists a map $\varphi: R^{+} \rightarrow R^{+}$continuous from the right for each $r \in R^{+}$such that

$$
d(T x, T y) \leq \varphi(d(x, y)) \text { for each } x, y \in X .
$$

If $\varphi(r)<r$ for $r>0$, then the sequence $\left\{T^{n}(x)\right\}_{n=0}^{\infty}$ converges to the unique fixed point of $T$.

In 1999, Nešić [6] proved the following theorem.

Theorem 1.7. Suppose $T$ is a selfmap of a metric space $(X, d)$ satisfying $d(T x, T y) \leq p d(x, y)+q[d(x, T x)+d(y, T y)]+r[d(x, T y)+d(y, T x)]+F(d(x, T x) . d(y, T y))$ 
for all $x, y$ in $X$, where $0 \leq p, r, p+2 r<1, q+r<1$. If $T$ is asymptotically regular at a point $x$ of $X$ and the sequence of iterates $\left\{T^{n} x\right\}$ has a subsequence converging to a point $z$ in $X$, then $z$ is the unique fixed point of $T$ and $\left\{T^{n} x\right\}$ also converges to $z$.

Example 1.8. Let $X=\left\{0,2^{-1}, 2^{-2}, 2^{-3}, \ldots\right\}$ with the usual metric. We define $T$ on $X$ by $T(0)=0, T\left\{2^{-n}\right\}=2^{-(n+1)}$ for $n=1,2,3, \ldots$. We define $F: R^{+} \rightarrow R^{+}$by $F(t)=t^{2}$. Then $T$ satisfies (1.7.1) with $p=2^{-1}, q=0, r=0 ; T$ is asymptotically regular at $2^{-1}$ and $T\left(2^{-n}\right)=2^{-(n+1)} \rightarrow 0$ as $n \rightarrow \infty$ so that $T$ satisfies all the hypotheses of Theorem 1.7; and ' 0 ' is the unique fixed point of $T$. Here we observe that $T$ is orbitally continuous at 0 .

The main aim of this paper is to avoid the ambiguity(established through Example 1.5) of Theorem 1.4 and generalize Theorem 1.6. Our theorem (Theorem 3.1) extends Theorem 1.6 to $T$-orbitally complete metric spaces. Also we prove a theorem (Theorem 3.6) by allowing the orbital continuity of $T$ at $z$ in Theorem 1.7. In this case the condition on $q$ in the inequality (1.7.1), i.e., $q+r<1$ is redundant, and can be relaxed.

Throughout this paper, we denote $R^{+}=[0, \infty)$, the set of all nonnegative reals and $N$, the set of all natural numbers and

$$
\Phi=\left\{\varphi:(0, \infty) \rightarrow(0, \infty) /(\mathrm{i}) \varphi(t)<t \text { for } t>0 \text {, and (ii) } \limsup _{t \rightarrow r^{+}} \varphi(t)<r \text { for } r>0\right\} .
$$

\section{Preliminaries}

Proposition 2.1. Let $T$ be a selfmap of a metric space $(X, d)$. If

(i) $T$ is asymptotically regular at some point $x_{0}$ of $X$,

(ii) $O\left(x_{0}\right)$ has a cluster point $z$ in $X$ and,

(iii) $T$ is orbitally continuous at $z$, then $z$ is a fixed point of $T$.

Proof. We define the sequence $\left\{x_{n}\right\}$ by $x_{n}=T^{n} x_{0}$ for $n=0,1,2, \ldots$, with $T^{0} x_{0}=$ $x_{0}$. If $x_{n}=x_{n+1}$ for some $n$, then $x_{m}=x_{n}$ for all $m \geq n$, so that $\lim _{m \rightarrow \infty} x_{m}=z$. Now by (iii), we have $T z=z$.

We now assume $x_{n} \neq x_{n+1}$ for all $n \in N$. Let $\{n(k)\}$ be the subsequence of positive integers such that $\left\{x_{n(k)}\right\}$ converges to $z$, by (ii). Using (i), we have $\lim _{k \rightarrow \infty} d\left(x_{n(k)}, x_{n(k)+1}\right)$ $=0$ and by (iii), it follows that $d(z, T z)=0$. Hence $T z=z$.

Example 2.2. Let $X=\{-1,0,1\} \cup\left\{2^{-k}: k \in N\right\} \cup\left\{1+2^{-k}: k \in N\right\}$ with the usual metric. We define $T: X \rightarrow X$ by $T(0)=-1 ; T(-1)=0$; for $x_{0}=1$, the sequence $\left\{x_{n}\right\}_{n=1}^{\infty}$ by $x_{n}=\left\{T^{n} x_{0}\right\}$ where $x_{2 n}=2^{-n}$ for $n=0,1,2, \ldots$, and $x_{2 n+1}=1+2^{-(n+1)}$ for $n=0,1,2,3, \ldots$, and

$$
O\left(x_{0}\right)=\left\{2^{-n}: n=0,1,2, \ldots\right\} \cup\left\{1+2^{-n}: n \in N\right\} .
$$


Also $x_{2 n}=2^{-n} \rightarrow 0$ as $n \rightarrow \infty$ and $T x_{2 n}=1+2^{-(n+1)} \rightarrow 1 \neq T(0)$ as $n \rightarrow \infty$ so that $T$ is not orbitally continuous at 0 . Also we observe that $T$ is not orbitally continuous at 1 , for $x_{2 n+1} \rightarrow 1$ and $T x_{2 n+1} \rightarrow 0 \neq T 1$ as $n \rightarrow \infty$. Also $T$ is not asymptotically regular at 1 ; and $T$ has no fixed point in $X$.

Example 2.3. Let

$$
X=\{1\} \cup\left\{\sum_{k=1}^{n} 2^{-k}: n \in N\right\}
$$

with usual metric. For $x_{0}=1$, we define

$$
x_{n+1}=T x_{n}=\sum_{k=1}^{n+1} 2^{-k} \text { for } n=0,1,2,3, \ldots
$$

Then $O\left(x_{0}\right)=X$, and has a cluster point $z=1$ in $X . T$ is asymptotically regular at $x_{0}=1$ and $T$ is not orbitally continuous at 1 . We observe that $T$ has no fixed point.

Example 2.4. Let $X=\{0,1\} \cup\left\{2^{-k}: k \in N\right\} \cup\left\{1+2^{-k}: k \in N\right\}$ with the usual metric. We define $T$ on $X$ by $T 0=1 ; T 1=0$ and for $x_{0}=1+2^{-1}$ we define the sequence $\left\{x_{n}\right\}_{n=1}^{\infty}$ by $x_{n}=T^{n} x_{0}$ where $x_{2 n}=1+2^{-(n+1)}$ and $x_{2 n-1}=2^{-n}$ for $n \in N$. Then $O\left(x_{0}\right)=X \backslash\{0,1\}$. Also $x_{2 n} \rightarrow 1$ as $n \rightarrow \infty$ and $T x_{2 n}=2^{-(n+1)} \rightarrow 0=T(1)$ as $n \rightarrow \infty$, so that $T$ is orbitally continuous at 1 . Also $T$ is not asymptotically regular at any point of $X$ and $T$ has no fixed point in $X$.

Example 2.5. Let $X=\{0,1\} \cup\left\{2^{-k}: k \in N\right\}$ with the usual metric. We define $T: X \rightarrow X$ by $T(0)=0, T(1)=2^{-1}, T\left(2^{-n}\right)=2^{-(n+1)}$ for $n \in N$. Then for $x_{0}=1$, $O\left(x_{0}\right)=\left\{2^{-n}: n=0,1,2,3, \ldots\right\}$ and $T$ satisfies all the conditions of the Proposition 2.1 and $T$ has a fixed point 0 .

Example 2.6. Let $X=\{0,1\} \cup\left\{2^{-k}: k \in N\right\} \cup\left\{1+2^{-k}: k \in N\right\}$ with the usual metric. We define $T$ on $X$ by $T 1=1 ; T 0=0 ; T\left(2^{-n}\right)=2^{-(n+1)}$ for $n \in N$ and $T\left(1+2^{-n}\right)=1+2^{-(n+1)}$ for $n \in N$; here $O\left(2^{-1}\right)=\left\{2^{-n}: n \in N\right\}$ and $O\left(1+2^{-1}\right)=$ $\left\{1+2^{-n}: n \in N\right\} . T$ is asymptotically regular at $2^{-1}$ and $1+2^{-1} ; O\left(2^{-1}\right)$ has a cluster point 0 and $O\left(1+2^{-1}\right)$ has a cluster point $1 ; T$ is orbitally continuous at 0 and 1 ; and $T$ has two fixed points 0 and 1.

\section{Main Results}

Theorem 3.1. Let $(X, d)$ be a T-orbitally complete metric space. Let $T$ be a selfmap of $X$. Assume that for some $x_{0} \in X$ there exists $\varphi_{x_{0}} \in \Phi$ such that

$$
d(T x, T y) \leq \varphi_{x_{0}}(d(x, y)) \text { for every } x \neq y \text {, and } x, y \in \overline{O\left(x_{0}\right)} .
$$

Then the sequence $\left\{x_{n}\right\}_{n=1}^{\infty}$ defined by $x_{n}=T^{n} x_{0}$ is Cauchy in $X, \lim _{n \rightarrow \infty} x_{n}=z$, $z \in X$ and if $T$ is orbitally continuous at $z$ then $z$ is a fixed point of $T$ and $z$ is unique in the sense that $\overline{O\left(x_{0}\right)}$ contains one and only one fixed point of $T$. 
Proof. We define the sequence $\left\{x_{n}\right\}$ by $x_{n}=T^{n} x_{0}$. If $x_{n}=x_{n+1}$ for some $n$, then we are through.

Hence, without loss of generality, we assume $x_{n} \neq x_{n+1}$ for all $n$. Let $a_{n}=$ $d\left(T^{n} x_{0}, T^{n+1} x_{0}\right)$. Now, by using (3.1.1) and property (i) of $\varphi_{x_{0}}$, we have

$$
a_{n+1}=d\left(T^{n+1} x_{0}, T^{n+2} x_{0}\right) \leq \varphi_{x_{0}}\left(d\left(T^{n} x_{0}, T^{n+1} x_{0}\right)\right)<d\left(T^{n} x_{0}, T^{n+1} x_{0}\right)=a_{n}
$$

so that $\left\{a_{n}\right\}$ is strictly decreasing sequence of reals. We now prove that $\lim _{n \rightarrow \infty} a_{n}=0$. Suppose $\lim _{n \rightarrow \infty} a_{n}=a>0$. Then by (ii) of $\varphi_{x_{0}}$, we have

$$
a=\limsup _{n \rightarrow \infty} a_{n+1} \leq \limsup _{n \rightarrow \infty} \varphi_{x_{0}}\left(a_{n}\right)<a,
$$

a contradiction. Therefore $\lim _{n \rightarrow \infty} a_{n}=0$.

Hence $T$ is asymptotically regular at $x_{0}$.

We now show that the sequence $\left\{x_{n}\right\} \subset O\left(x_{o}\right)$ is Cauchy. Otherwise, there is an $\epsilon>0$ and there exist sequences $\{m(k)\}$ and $\{n(k)\}$ with $m(k)>n(k)>k$ such that

$$
d\left(x_{m(k)}, x_{n(k)}\right) \geq \epsilon \quad \text { and } \quad d\left(x_{n(k)-1}, x_{m(k)}\right)<\epsilon .
$$

Hence

$$
\epsilon \leq d\left(x_{m(k)}, x_{n(k)}\right) \leq d\left(x_{m(k)}, x_{m(k)-1}\right)+d\left(x_{m(k)-1}, x_{n(k)}\right)<a_{m(k)-1}+\epsilon .
$$

By taking limits as $k \rightarrow \infty$, we have

$$
\lim _{k \rightarrow \infty} d\left(x_{m(k)}, x_{n(k)}\right)=\epsilon .
$$

Also

$$
\begin{aligned}
\epsilon \leq d\left(x_{m(k)}, x_{n(k)}\right) & \leq \varphi_{x_{0}}\left(d\left(x_{m(k)-1}, x_{n(k)-1)}\right)\right) \\
& <d\left(x_{m(k)-1}, x_{n(k)-1}\right) \\
& \leq d\left(x_{m(k)-1}, x_{m(k)}\right)+d\left(x_{m(k)}, x_{n(k)}\right)+d\left(x_{n(k)}, x_{n(k)-1}\right) \\
& =a_{m(k)-1}+d\left(x_{m(k)}, x_{n(k)}\right)+a_{n(k)-1} .
\end{aligned}
$$

Now, taking limits as $k \rightarrow \infty$, we have

$$
\epsilon \leq \lim _{k \rightarrow \infty} d\left(x_{m(k)-1}, x_{n(k)-1}\right) \leq \lim _{k \rightarrow \infty} d\left(x_{m(k)}, x_{n(k)}\right)=\epsilon
$$

so that

$$
\lim _{k \rightarrow \infty} d\left(x_{m(k)-1}, x_{n(k)-1}\right)=\epsilon .
$$

Therefore, using (3.1.3), (3.1.4) and property (ii) of $\varphi_{x_{0}}$, we have

$$
\epsilon=\limsup _{k \rightarrow \infty} d\left(x_{m(k)}, x_{n(k)}\right) \leq \limsup _{k \rightarrow \infty} \varphi_{x_{0}}\left(d\left(x_{m(k)-1}, x_{n(k)-1}\right)\right)<\epsilon,
$$


a contradiction. Hence $\left\{x_{n}\right\} \subset O\left(x_{o}\right)$ is Cauchy. Since $X$ is $T$-orbitally complete,

$$
\lim _{n \rightarrow \infty} x_{n}=z \text { (say), } z \in X .
$$

Since $T$ is orbitally continuous at $z$, using (3.1.2) and (3.1.5) and by Proposition 2.1, it follows that $z$ is a fixed point of $T$.

Uniqueness of the fixed point trivially follows from (3.1.1).

This completes the proof of the theorem.

Remark 3.2. In the following we provide an example for the applicability of Theorem 3.1 where as Theorem 1.6 is not applicable.

Example 3.3. Let

$$
X=\{0,1,2\} \cup\left\{\sum_{i=0}^{n} 2^{-i} ; n \in N\right\} .
$$

We define $T$ on $X$ by $T 0=1 ; T 1=0 ; T 2=2$; and

$$
T\left(\sum_{i=0}^{n} 2^{-i}\right)=\sum_{i=0}^{n+1} 2^{-i} \quad \text { for } n \in N .
$$

Clearly, $X$ is $T$-orbitally complete metric space and $T$ is orbitally continuous at 2 .

At $x=0 ; y=1$; for any $\varphi \in \Phi$, the condition (1.6.1) does not hold.

If $x_{0}=1+2^{-1}$ then

$$
O\left(x_{0}\right)=\left\{\sum_{i=0}^{n} 2^{-i}: n \in N\right\}, \quad \overline{O\left(x_{0}\right)}=O\left(x_{0}\right) \cup\{2\}
$$

and $T$ satisfies all the hypotheses of Theorem 3.1 with $\varphi(t)=2^{-1} t$, for all $t>0$; and 2 is the unique fixed point of $T$.

Remark 3.4. Under the hypotheses of Theorem 3.1, $T$ may have more than one fixed point in $X$. For example, we consider the space $X$ as in Example 2.6. We define $T$ on $X$ by $T 1=1, T 0=0$,

$$
T\left(2^{-n}\right)=2^{-(n+1)}, T\left(1+2^{-n}\right)=1+2^{-(n+1)} \text { for } n \in N .
$$

If $x_{0}=2^{-1}$ then $O\left(2^{-1}\right)=\left\{2^{-n}: n \in N\right\}$ and $\overline{O\left(2^{-1}\right)}=O\left(2^{-1}\right) \cup\{0\} . T$ satisfies all the hypotheses of Theorem 3.1 with $\varphi_{2^{-1}}(t)=t(1+t)^{-1}, t>0$ and ' 0 ' is a fixed point of $T$.

If $x_{0}=1+2^{-1}$, then $O\left(1+2^{-1}\right)=\left\{1+2^{-n}: n \in N\right\}$ and $\overline{O\left(1+2^{-1}\right)}=O\left(1+2^{-1}\right) \cup$ $\{1\}$. Then $T$ satisfies all the conditions of Theorem 3.1 with $\varphi_{\left(1+2^{-1}\right)}(t)=2^{-1} t, t>0$ and 1 is another fixed point of $T$.

We observe that $T$ has two fixed points 0 and 1 . 
Remark 3.5. We extend Theorem 1.6 of Mukherjee to $T$-orbitally complete metric spaces in the following way.

Theorem 3.6. Let $(X, d)$ be a $T$-orbitally complete metric space. Let $T$ be a selfmap of $X$. Assume that there exists $\varphi \in \Phi$ such that

$$
d(T x, T y) \leq \varphi(d(x, y)), \text { for each } x \neq y, x, y \in X .
$$

Then, for $x_{0} \in X$, the sequence $\left\{x_{n}\right\}_{n=1}^{\infty}$ defined by $x_{n}=T^{n} x_{0}$ is Cauchy in $X$, $\lim _{n \rightarrow \infty} x_{n}=z, z \in X, z$ is the unique fixed point of $T$ provided $T$ is orbitally continuous at $z$.

We now generalize Theorem 1.7 of Nešić in the following way.

Theorem 3.7. Suppose that $T$ is a selfmap of a metric space $(X, d)$. Assume that there exist non-negative real numbers $p$, $q$ and $r$ such that

$d(T x, T y) \leq p d(x, y)+q[d(x, T x)+d(y, T y)]+r[d(x, T y)+d(y, T x)]+F(d(x, T x) . d(y, T y))$

for all $x, y \in X$ and $p+2 r<1$.

If $T$ satisfies (i), (ii), (iii) of Proposition 2.1, then $z$ is the unique fixed point of $T$ and for any $x_{0} \in X$ the sequence $\left\{T^{n} x_{0}\right\}$ converges to $z$.

Proof. Existence of a fixed point of $T$ follows from Proposition 2.1. Uniqueness of fixed point follows from the inequality (3.7.1). Let that fixed point be $z$.

We now show that $\lim _{n \rightarrow \infty} x_{n}=z$. Let $x_{0} \in X$. We consider

$$
\begin{aligned}
d\left(z, T^{n} x_{0}\right)= & d\left(T z, T^{n} x_{0}\right) \\
\leq & d\left(T z, T^{n+1} x_{0}\right)+d\left(T^{n+1} x_{0}, T^{n} x_{0}\right) \\
\leq & p d\left(z, T^{n} x_{0}\right)+q\left[d(z, T z)+d\left(T^{n} x_{0}, T^{n+1} x_{0}\right)+r\left[d\left(z, T^{n+1} x_{0}\right)\right.\right. \\
& \left.+d\left(T^{n} x_{0}, T z\right)\right]+F\left(d(z, T z) \cdot d\left(T^{n} x_{0}, T^{n+1} x_{0}\right)\right)+d\left(T^{n} x_{0}, T^{n+1} x_{0}\right) \\
\leq & p d\left(z, T^{n} x_{0}\right)+q d\left(T^{n} x_{0}, T^{n+1} x_{0}\right)+r\left[d\left(z, T^{n} x_{0}\right)\right. \\
& \left.+d\left(T^{n} x_{0}, T^{n+1} x_{0}\right)+d\left(T^{n} x_{0}, z\right)\right]+d\left(T^{n} x_{0}, T^{n+1} x_{0}\right)
\end{aligned}
$$

(by using $T z=z$ ).

Hence

$$
d\left(z, T^{n} x_{0}\right) \leq(1+q+r)(1-p-2 r)^{-1} d\left(T^{n} x_{0}, T^{n+1} x_{0}\right) .
$$

Taking limits as $n \rightarrow \infty$, by using (i) of Proposition 2.1, and since $p+2 r<1$, it follows that $\lim _{n \rightarrow \infty} x_{n}=z$.

This completes the proof of this theorem.

Example 3.8. Let $X$ be as in the Example 3.3. Now, we define $F$ be the identity mapping on $R^{+}$. Let $x_{0}=1+2^{-1}$ then

$$
O\left(x_{0}\right)=\left\{T^{n} x_{0}=\sum_{k=0}^{n+1} 2^{-k}: n \in N\right\} \cup\left\{1+2^{-1}\right\} .
$$


Clearly $T$ is asymptotically regular at $x_{0}, T$ satisfies the inequality (3.7.1) with $p=0$; $r=3^{-1}$ so that $p+2 r<1$ and $q=3 ; O\left(x_{0}\right)$ has a cluster point 2 ; and $T$ is orbitally continuous at 2; so that $T$ satisfies all the hypotheses of Theorem 3.7 and $T$ has the unique fixed point 2 .

We observe that if we choose $q=0$ then for $x=1$ and $y=2$, the inequality (1.7.1) does not satisfied for any non-negative reals $p$ and $r$ with $p+2 r<1$, and hence Nešić's theorem (Theorem 1.7) is not applicable.

\section{Acknowledgements}

This work is supported by UGC Grant No. F. 8-8/2003 (SR). The authors thank the University Grants Commission, India.

\section{References}

[1] G. V. R. Babu, Generalization of fixed point theorems relating to the diameter of orbits by using a control function, Tamkang J. of Math. 35 (2004), 159-168.

[2] F. E. Browder and W. V. Petryshyn, Construction of fixed points of nonlinear mappings in Hilberts spaces, J. Math. Anal. Appl. 20(1967), 197-228.

[3] Lj. B. Ćrić, A generalization of Banach's contraction principle, Proc. Amer. Math. Soc., 45 (1974), 267-273.

[4] A. Mukherjee, Contractions and completely continuous mappings, Nonlinear Analysis, TMA 1 (1977), 235-247.

[5] Nikola Jotic, Some fixed point theorems in metric spaces, Indian J. Pure Appl. Math., 26 (1995), 947-952.

[6] Slobodan C. Nešić, Results on fixed points of asymptotically regular mappings, Indian. J. Pure Appl. Math., 30 (1999), 491-494.

Department of Mathematics, Andhra University, Visakhapatnam 530 003, India.

E-mail: gvr_babu@hotmail.com 